\title{
Análisis de la estrategia de marketing digital mediante herramientas de analítica web
}

\author{
Vilma Álvarez Intriago / Luis Agreda Fernández
}

Antonio Cevallos Gamboa

\section{Resumen}

El objetivo del presente artículo es determinar el impacto de la estrategia de marketing digital en el tráfico y en el comportamiento de los usuarios hacia el sitio web de una escuela de postgrado en el Ecuador. Para ello, mediante el uso de las herramientas Google Analitycs y Piwik, se examinan las redes sociales Facebook y Twitter. En este contexto, se concluye que cuando se realiza una estrategia de marketing digital en las redes sociales se obtiene un impacto positivo en el tráfico y comportamiento de los usuarios hacia el sitio web.

\section{Palabras clave:}

Analitica web, Google analytics, Piwik, Métricas, Redes sociales.

\section{Abstract}

This article aims to ascertain traffic and user-behavior impact of digital marketing strategy on an Ecuadorian postgraduate school's website. For that purpose, in order to examine Facebook and Twitter, the tools used were Google Analytics and Piwik: It was concluded that, when a digital marketing strategy is used in social networks, a positive traffic and user behavior impact toward the website is obtained.

Keywords:

Web analytics, Google analytics, Piwik, Metrics, Social networks. 


\section{Introducción}

Las redes sociales en las empresas, han mejorado sus condiciones, oportunidades, rentabilidad y permanencia en el mercado, permitiéndole alcanzar eficientemente sus objetivos y ejecutar eficazmente sus estrategias de marketing (Lamas, 2015).

Sanjaime (2012) analiza el grado de generalización del uso de las redes sociales como parte de las estrategias de marketing, recopilando estudios realizados por diferentes empresas e instituciones españolas e internacionales. En el mismo, se examina el conjunto de técnicas y herramientas más utilizadas en el ámbito del marketing en redes sociales y los elementos relacionados con la gestión de la presencia de marca de las empresas.

Uribe (2010) expresa que las redes sociales digitales son un fenómeno global y creciente, que permiten la comunicación entre personas que hace algunos años atrás no era posible. En este sentido, se identifican dos nuevos aspectos: la analítica web, que hace referencia al análisis de los datos que son recogidos en internet y que brindan asistencia en la gestión de una empresa y la optimización mediante una estrategia digital. Es asi que el presente estudio se basa en el análisis de los aspectos antes mencionados (Morley, 2015).

Por otra parte, la escuela de posgrados, que fue creada con la visión de ser la escuela de negocios líder en Ecuador y constituirse como referente de opinión nacional e internacional en los ámbitos empresarial, social, político y económico, actualmente cuenta con un centro de desarrollo de emprendedores, que da referencia de documentos de trabajo y casos de emprendedores ecuatorianos. El eje de su negocio es la educación de cuarto nivel, ofertando maestrías MBA \& EMBA y maestrías especializadas en Gerencia Hospitalaria y Gestión de Proyectos. Cuenta con un gran portafolio de programas complementarios para la formación de ejecutivos principalmente en el área de Negocios.

En tal virtud, se sugiere la iniciativa de que en la escuela de posgrados se establezca una campaña de información masiva por medio de las redes sociales, que permita incrementar el número de usuarios que accedan a la Web, en busca de la oferta académica brindada por la institución. (Degiorgi \& Mendez, 2013).

Al no conocer datos numéricos sobre el desempeño de las redes sociales en el marketing digital, surge la necesidad de establecer métricas que permitan determinar la efectividad de esta estrategia (Agüera, 2014).

Bajo este contexto, se realiza el análisis del impacto de las redes sociales facebook y twitter a través de dos herramientas: google analytics y piwik, implementadas dentro de una estrategia de marketing digital, con el propósito de demostrar, que estos mecanismos de difusión, contribuyen en el manejo efectivo de la comunicación directa en una institución de educación superior (Plaza, 2009). Se comenzará con la conceptualización de términos relevantes; posteriormente se realizará la selección de métricas y el análisis de los resultados obtenidos en las dos herramientas utilizadas.

\section{Marco teórico}

\section{Marketing digital}

Olmo y Gascón (2014) definen al marketing digital como una nueva área de negocios vinculada al desarrollo del Internet. Si una marca no está en la web no existe, de ahí se derivan los nuevos puestos de trabajo como Community Manager y Social Media Manager. Por su parte, las redes sociales Facebook, Twitter, Instagram, YouTube y otras, son canales digitales de información y cumplen una función 
muy importante en la difusión de la marca o productos. No obstante, toda campaña de marketing digital, debe tener el apoyo de un sitio web que debe estar enlazado a todos los canales sociales de la empresa (Sheehan, 2010).

Gálvez (2010) menciona que el realizar una planificación, permite minimizar los riesgos de la empresa ante situaciones imprevistas. La planificación tiene como beneficio el aporte de una línea estratégica, priorización de asignación de recursos, optimización de procesos organizativos, base de control y la identificación, tanto de los problemas que se puede presentar, como de las posibles oportunidades de una organización.

El marketing digital se constituye en la gestión que hace una empresa para dar a conocer, promover y vender productos y servicios por internet (Kotler \& Armstrong, 2008). En término general el marketing digital tiene como finalidad aprovechar las oportunidades que brinda internet para potenciar un negocio de una manera más eficiente (García \& Aguado, 2009).

\section{Redes Sociales}

Campos (2008) afirma que el rápido y exitoso debut en los dos o tres últimos años de las llamadas redes sociales han encendido las alarmas en los medios de comunicación tradicionales.

Brunetta (2013) asegura que el crecimiento de las redes sociales ha motivado a que las empresas adopten un nuevo esquema informativo, basado en el análisis de las impresiones de los usuarios, como un aspecto fundamental para el éxito empresarial. Los usuarios ingresan a las redes sociales buscando información, marcas y productos ofrecidos por las empresas. Sin embargo, Caldevilla (2010) menciona que las redes sociales dan origen a una discusión en torno a la privacidad y a los peligros de estas nuevas formas de interacción.

\section{Analítica web}

Maldonado (2010) menciona que analítica web es una disciplina que permite extraer conclusiones, establecer reglas de negocio o definir estrategias basándose en los datos que son obtenidos de los sitios web, sobre los que una empresa basa su propuesta de marketing digital. El mencionar a la analítica web como una herramienta de marketing digital no está alejado de la verdad, debido a que se basa en recoger datos que se producen en el tráfico web, para posteriormente realizar su análisis $e$ interpretación (Villaplana, 2013a; Villaplana, 2013b; Villaplana, 2014).

Digital Analytics Association (2015) define a la analítica Web como una medición, recogida, análisis e interpretación de los datos de tráfico web que tiene por objetivo entender y optimizar la navegación web" (pág. 1). Esta herramienta de análisis ayuda a conocer a los usuarios, quiénes son, dónde están viendo y cómo utilizan su sitio web (Olguin \& Klenzi, 2012).

Según lo evidenciado por los anteriores autores, las empresas que prescinden de las redes sociales apoyadas por herramientas de analítica web, equivalen a un negocio cerrado. Las redes sociales se han transformado en una herramienta importante dentro de una estrategia de marketing digital (Morales, 2010).

\section{Métricas y estándares}

Fernández-Villavicencio (2013) propone que la recolección de datos del tráfico generado hacia un sitio web o redes sociales, se puede medir no solo a partir de los visitantes únicos, sino también de los sitios vistos, el tiempo de permanencia o la tasa de rebote. 
Figura 1.

Métricas básicas y su descripción.

\begin{tabular}{|c|c|}
\hline Páginas vistas & $\begin{array}{c}\text { - Hace referencia al número de veces que un usuario accede a una } \\
\text { página del sitio web por sesión. }\end{array}$ \\
\hline Visitas & $\begin{array}{c}\text { - Se refiere al tiempo de interacción entre el navegador y un sitio } \\
\text { web. }\end{array}$ \\
\hline Visitantes únicos & $\begin{array}{c}\text { - Permite conocer el número de usuarios que han visitado el sitio } \\
\text { web durante un determinado periodo. }\end{array}$ \\
\hline Tiempo de estancia & $\begin{array}{c}\text { - Corresponde al periodo de tiempo de todas las visitas en la } \\
\text { página web sobre el número de visitas realizadas. }\end{array}$ \\
\hline Tasa de rebote & $\begin{array}{c}\text { - Permite definir el porcentaje de las visitas en el sitio web que } \\
\text { permanecieron solo unos pocos segundos y lo abandonaron. }\end{array}$ \\
\hline Tasa de salida & - Se refiere al porcentaje de visitas que abandonaron el sitio web \\
\hline
\end{tabular}

Fuente: Los autores

Martínez, (2013) recomienda que para comparar el tráfico de diversos sitios web existen métricas básicas que se pueden utilizar para trabajar con herramientas de analítica web, como las páginas visitadas, navegadores únicos y las visitas al sitio web. Cuando un usuario accede a una página web a través de un browser, una cookie identifica este navegador. A continuación en la Figura 1 se presentan algunas métricas básicas y su descripción.

\section{Piwik}

Piwik (2015) es una plataforma Open Source, líder en el manejo de la analítica web, que brinda la posibilidad de obtener información valiosa para las empresas como: los visitantes, el funcionamiento de las estrategias de marketing, entre otros, para poder optimizar una campaña online y la experiencia de los usuarios. Piwik brinda la posibilidad de obtener información detallada sobre las visitas en un sitio web; así como la descarga de documentos, las páginas visitadas, los navegadores, el tráfico en el sitio web.
Miller (2015) menciona que la principal ventaja de Piwik es la privacidad y control absoluto de los datos, esto debido a que la instalación se la realiza en el propio servidor sin depender de fuentes externas. Además tiene múltiples funcionalidades de administración, como la gestión de usuarios, rangos IP, parámetros URL y mantenimiento automático de la base de datos.

\section{Google Analytics}

Lebrón (2011) indica que con la utilización de esta herramienta se puede responder preguntas tales como: ¿cuál es el posicionamiento?, ¿el sitio web es fácil de usar?, ¿de dónde provienen las visitas?, ¿funciona la publicidad en la web?, ¿cuánto tiempo se quedan los usuarios?, entre otras. En sintesis, es una herramienta web que proporciona a las empresas información sobre el tráfico del sitio web y ayuda a medir la eficacia de la estrategia de marketing (Hasan, Morris \& Probets, 2009). 
Pérez (2013) menciona que Google Analitycs es una herramienta libre que proporciona información sobre estadísticas de accesos de un sitio web. A pesar de que no fue diseñada para redes sociales, ayuda a descubrir su efectividad, porque permite contabilizar los accesos de entrada directa, separando los que acceden a través de los buscadores y de los que acceden a través de las redes sociales (Hess, 2012).

\section{Metodología}

La investigación es de carácter exploratorio y está formulada con un perfil analítico-descriptivo, por lo cual se considera una fundamentación teórica que sustenta el argumento a evaluar a traves del uso de herramientas de analítica web. En este sentido, se plantean cinco fases de estudio:

a. Definición de objetivos y KPIs: Permite definir los objetivos concretos que se esperaban alcanzar y que fueron caracterizados por ser SMART. La definición de KPIs sirve para lograr una visión correcta sobre la situación actual del negocio.

b. Implementación y medición: La implementación avanzada de las herramientas de la analítica web, permite obtener datos relevantes para el negocio; la correcta implementación de las herramientas, asegura la calidad de los datos. Para este proceso se utilizaron dos herramientas de medición Google Analytics y Piwik.

c. Reporte: En la etapa de reporte se cuenta con una interfaz de usuario personalizada, esto facilita la correcta toma de decisiones, que se la hace en base a la información mostrada por los KPIs de una manera muy dinámica y sencilla.

d. Análisis: En la metodología de analítica web la etapa de análisis corresponde a un área muy compleja. Se realiza basándose en los objetivos descritos inicialmente y los datos obtenidos luego de la aplicación de una estrategia digital.

e. Optimización: Una vez obtenidos los resultados del análisis, se puede establecer procesos o estrategias enfocados en la mejora del sitio web.

Finalmente, para el analisis se procede a la implementación de las herramientas para la obtención de los datos de forma simultánea, con el fin de obtener una visión clara sobre el funcionamiento, tanto de piwik como de google analytics. Para ello, con el objeto de realizar un análisis comparativo de la información brindada por las herramientas en el estudio, los datos que se mostrarán corresponden al periodo comprendido entre el 15 de agosto y el 15 de septiembre del 2015.

Analisis y presentación de resultados

Utilizando las herramientas de analítica web en sus diferentes etapas, los resultados obtenidos son los siguientes:

a. Definición de objetivos y KPIs

Se establecieron aspectos referentes a las métricas que operan en las redes: interacción, comunicación entre usuarios y red social; acción, nivel de motivación para interactuar en base a los contenidos; alcance, difusión de información y aceptación y satisfacción de los usuarios.

De la aplicación de lo antes mencionado, se pudo obtener indicadores que se relacionan con la empresa y sus objetivos. Sin embargo, las redes sociales mantuvieron sus propios indicadores que pudieron ser utilizados para generar informes y permitieron dar seguimiento a las estrategias. En la Tabla 1 se muestra un detalle de las métricas: interacción, la comunicación entre usuarios y red social; acción, nivel de motivación para interactuar en base a los contenidos; alcance, difusión de información, aceptación y satisfacción de los usuarios 


\begin{tabular}{|c|c|c|c|}
\hline \multicolumn{3}{|l|}{ Tabla \# 1} & \\
\hline \multicolumn{4}{|c|}{ Indicadores en las redes sociales Facebook y Twitter } \\
\hline \multirow{2}{*}{\multicolumn{2}{|c|}{ Análisis }} & \multicolumn{2}{|c|}{ Indicadores } \\
\hline & & Facebook & Twitter \\
\hline \multirow{3}{*}{\multicolumn{2}{|c|}{ Interacciones }} & Comentarios & Menciones \\
\hline & & Compartir & Retweet \\
\hline & & Me gusta & Clics en enlaces \\
\hline \multirow{2}{*}{\multicolumn{2}{|c|}{ Audiencia }} & Fans & Seguidores \\
\hline & & Publicaciones & Tweets \\
\hline \multirow{10}{*}{ Compromiso } & \multirow{2}{*}{ Conversación } & Comentarios & Menciones \\
\hline & & Fans & Seguidores \\
\hline & \multirow{2}{*}{ Amplificación } & Compartir & Retweet \\
\hline & & Fans & Seguidores \\
\hline & \multirow{2}{*}{ Acción } & & Clic enlace \\
\hline & & & Seguidores \\
\hline & \multirow{2}{*}{ Aceptación } & Me gusta & \\
\hline & & Fans & \\
\hline & \multirow{2}{*}{ Interacciones } & Comentarios+Compartir+Me gusta & Menciones+Retweet \\
\hline & & Fans & Seguidores \\
\hline \multirow{10}{*}{ Interés } & \multirow{2}{*}{ Conversación } & Comentarios & Menciones \\
\hline & & Fans & Tweet \\
\hline & \multirow{2}{*}{ Amplificación } & Compartir & Retweet \\
\hline & & Publicaciones & Tweet \\
\hline & \multirow{2}{*}{ Acción } & Clic enlace & Clic enlace \\
\hline & & Publicaciones & Tweet \\
\hline & \multirow{2}{*}{ Aceptación } & Me gusta & \\
\hline & & Publicaciones & \\
\hline & \multirow{2}{*}{ Interacciones } & Comentarios+Compartir+Me gusta & Menciones+Retweet \\
\hline & & Publicaciones & Tweet \\
\hline
\end{tabular}

Fuente: Decreto Ejecutivo 1215. (2 de febrero del 2001). Reglamento Sustitutivo del Reglamento Ambiental para las Operaciones Hidrocarburiferas en el Ecuador. Quito, Ecuador.

Mediante los indicadores del sitio web se pudo obtener el porcentaje de visitas, número de visitas, visitantes nuevos y recurrentes, duración de la visita, páginas vistas, porcentaje de rebote, ubicación geográfica de los visitantes, navegadores, dispositivos, sistemas operativos, entre otros aspectos, que ayudaron a entender el comportamiento de los usuarios. Para esto, fue necesario definir las métricas que serían utilizadas para dar seguimiento a los medios, con el propósito de realizar cambios que permitieron mejorar el negocio.
Así, el primer objetivo estuvo orientado a dar respuesta a los clientes ofreciendo los servicios que las maestrías de la escuela de posgrado promocionan. Mientras que el segundo objetivo se orientó a vender el producto, haciendo referencia a la venta como un aspecto fundamental de un negocio. Luego el tercer objetivo estuvo enfocado a incrementar y mantener la fidelidad de los usuarios. Finalmente, el cuarto objetivo persiguió el posicionamiento de la marca en la web, destacando el interés de los usuarios. 


\section{b. Implementación y medición.}

Para esta etapa del proceso se hizo uso de dos herramientas de medición: Piwik y Google Analytics.

Para comenzar el análisis con Piwik, luego de realizar el proceso de instalación en el servidor, fue preciso agregar líneas de código en el sitio web de la escuela de posgrado. En la Figura 2, se observa el código necesario para el estudio.

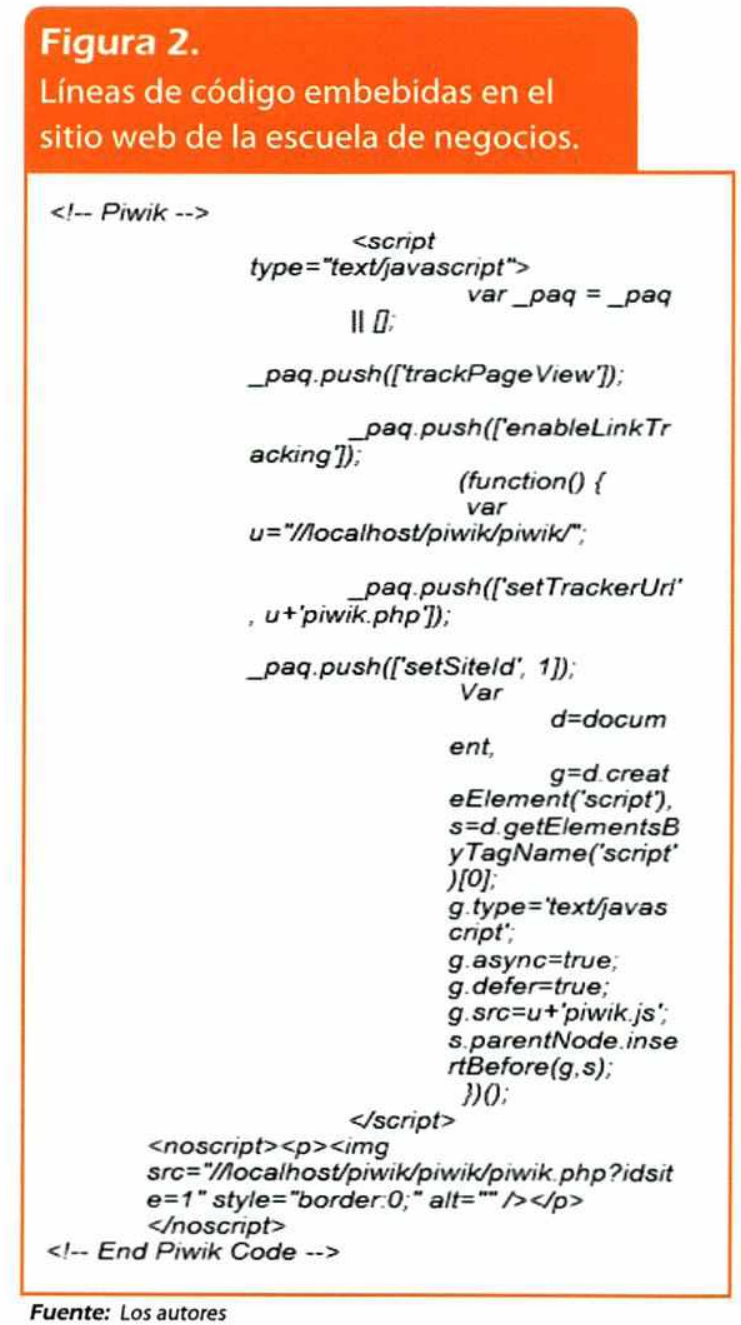

El código de seguimiento de Piwik se puede obtener iniciando la sesión como administrador. En el menú ajustes, opción "obtener el código de seguimiento" generado automáticamente por la aplicación instalada en el servidor. En la Figura 3, se puede observar el código de seguimiento de Piwik.

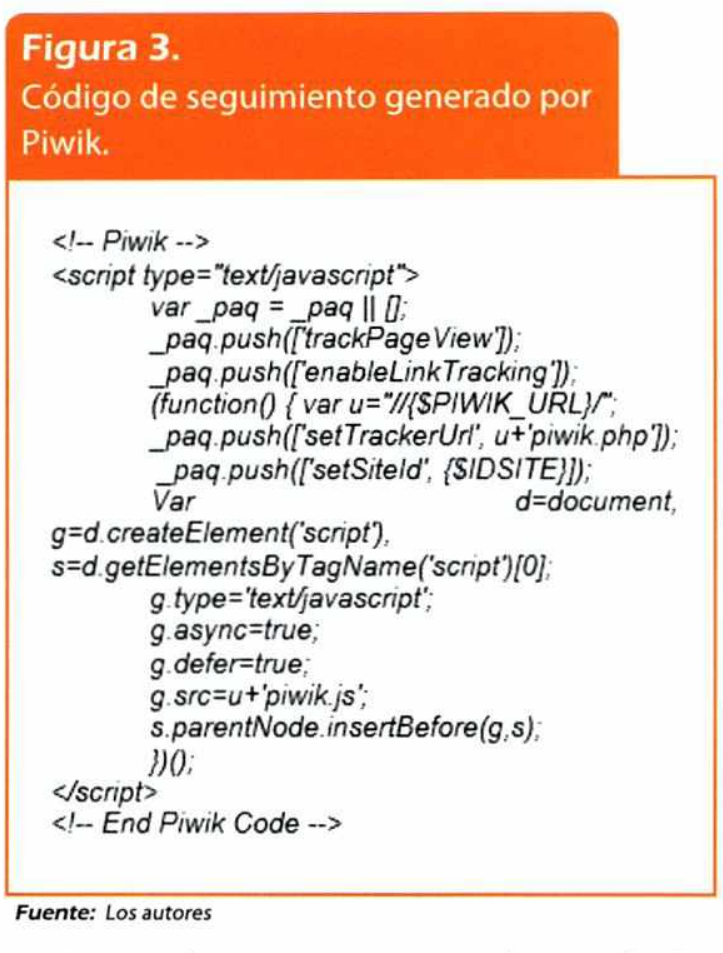

Se puede mencionar que dentro de los aspectos más importantes el $\{$ PIWIK_URL\} debió ser reemplazado por el URL correspondiente al sitio web en la variable u; de igual manera reemplazar el id Piwik del sitio web por $\{$ IDSITE\}. Para realizar el seguimiento asíncrono, las llamadas de conexión son enviadas a la matriz global_paq para la ejecución, independiente de la carga asíncrona de piwik.js. En la siguiente línea de código podemos observar el formato utilizado: _paq.push (['API_method_name', parameter_list]);

Luego se personaliza el nombre de la página dentro de la librería Piwik.js, la cual es una característica de seguimiento de JavaScript. Piwik utiliza la URL de la página actual y se puede personalizar mediante el siguiente, dos líneas de código: _paq.push(['setDocumentTitle', document. title]); y_paq.push(['trackPage View']);

Además se realiza la activación de forma manual de los eventos. Por defecto se carga el código de seguimiento cuando Piwik rastrea las páginas vistas y se ejecuta en cada una de las páginas vistas. Piwik permite realizar el seguimiento de las 
interacciones de los usuarios manualmente invocando a la función trackEvent(), escribiendo las siguientes líneas de código: $<$ a href="\#" onclick="javascript:_paq.push (['trackEvent', 'Menu', 'Freedom']);c"> Enlace</a $>$.

Piwik también permite activar manualmente las conversiones de objetivos, los que se definen como partes iguales de la dirección URL. También puede realizar un seguimiento de las metas para páginas vistas, descargas, o clics fuera del sitio. De igual manera las conversiones de un usuario se pueden cambiar cuando ha permanecido mucho tiempo en la página, o cuando se envía un formulario, entre otros. De tal manera, la sentencia _paq.push (['trackGoal ", 1]); registra una conversión para la meta 1 .

Para el seguimiento de búsqueda interna la librería Piwik.js ofrece una avanzada función de análisis en el sitio web, esto permite realizar un seguimiento de cómo los usuarios o visitantes usan su motor de búsqueda web interno en el sitio. Piwik puede leer parámetros de URL que contendrán la palabra clave de búsqueda, también puede grabar la palabra clave de búsqueda del sitio de forma manual utilizando la función Javascript trackSiteSearch ().

Así, para mantener un seguimiento de número de visitas, Piwik utiliza cookies de origen donde el ID del visitante es único. Las cookies se caracterizan por garantizar mayor privacidad de los usuarios y por lo tanto son aceptadas en la mayoría de los navegadores por defecto.

Entonces, Piwik en sus procesos crea un conjunto de cookies para cada dominio y subdominio. $\mathrm{Si}$ desea realizar un seguimiento de algunos subdominios y compartir la misma cookie de estadísticas precisas, es necesario personalizar el código de seguimiento de Piwik.

\section{Instalación y conexión de Piwik}

La instalación de Piwik se la realizó en un servidor local de la escuela de negocios. Como primer paso se descargaron las librerías de Piwik de la siguiente URL: http://piwik.org/download/.

Luego de descargar la librería Piwik se ingresó al servidor de aplicaciones web y se realizó la instalación. Una vez ingresado al panel de administración del hosting, se procedió a crear la base de datos en MySql.

Después de la creación de la base de datos se subió el paquete de Piwik al hosting para guardar los registros y que estén disponibles para su posterior análisis. Se puede utilizar cualquier cliente FTP. En este caso se utilizó el administrador de archivos del hosting (FileZilla). Inmediatamente, se procedió a la instalación de las librerías, accediendo a la página de instalación por medio del domino, seguido por el nombre de la carpeta Piwik.

Para la administración de Piwik se requirió la creación de un súper usuario y la conexión del sitio web de análisis. Una vez conectados estos campos la librería Piwik.js generó un código de seguimiento, el mismo que debió ser insertado en las etiquetas: $<$ header $><$ /header $>0<$ footer $><$ /footer $>$ del sitio web de la escuela de postgrado del Ecuador.

Google Analytics: Recopilación de datos por medio de Google Analytics.

Esta herramienta trabaja con código JavaScript, para efecto del análisis se deben insertar líneas de código en la página web de la escuela de negocios y así obtener los datos. En la Figura 4 se muestra el código mencionado. 


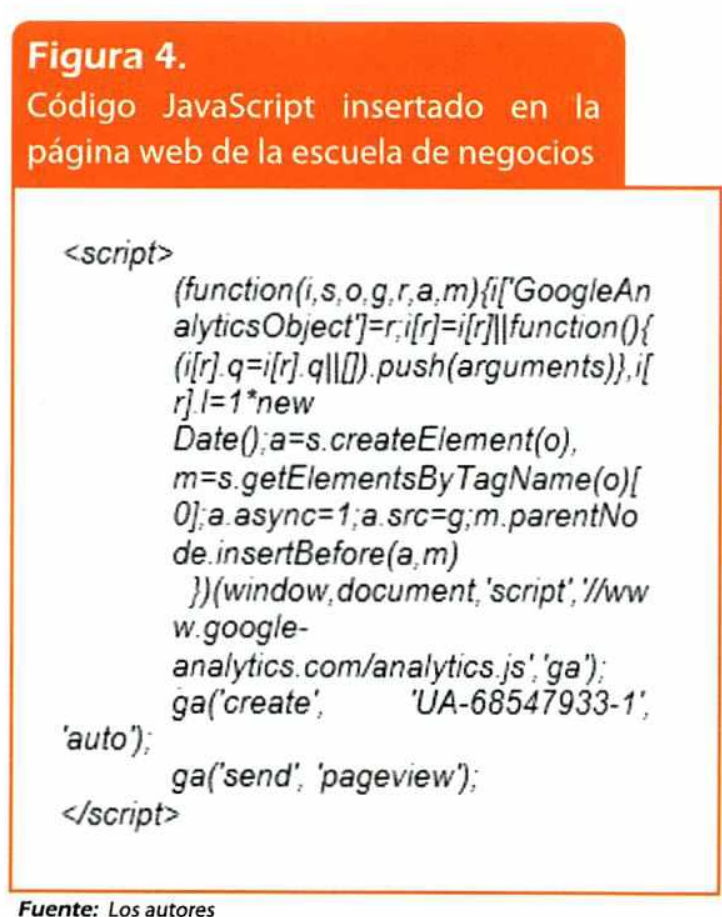

Mediante el Google Analytics Tracking Code [GACT], se realiza un proceso de carga de un archivo desde el servidor de Google, asignándole variables al número de cuenta de cada uno de los usuarios.

El GATC utilizó cookies para guardar la información de los usuarios, también almacenó aspectos como reconocer un nuevo usuario, la sesión del usuario, la ubicación, navegadores, entre otros. Cabe resaltar que el código generado fue ingresado en cada una de la página antes mencionada. Como la página web de la escuela de negocios fue desarrollada en el lenguaje de programación PHP, se pudo crear un archivo con extensión php llamado analyticstracking e incluirlo en cada una de las páginas donde se realizó el estudio. A continuación se indica la porción de código que se incluyó: php include_once ("analyticstracking.php") ? >

\section{Análisis del código de Google Analytics}

Puesto que Google Analytics utiliza el lenguaje JavaScript con sus respectivas librerias para su funcionamiento. Se utilizó la librería Analytics.js para realizar un análisis de cómo interactúan los usuarios en un determinado sitio web. En la Figura 5 se muestra el fragmento de código JavaScript que se insertó para el seguimiento en las plantillas del sitio web de la escuela de postgrado del Ecuador, dentro de las etiquetas $<$ head $><$ /head $>$ o $<$ footer $><$ /footer $>$.

\section{Figura 5.}

Código de seguimiento insertado en las plantillas del sitio web de la escuela de negocio

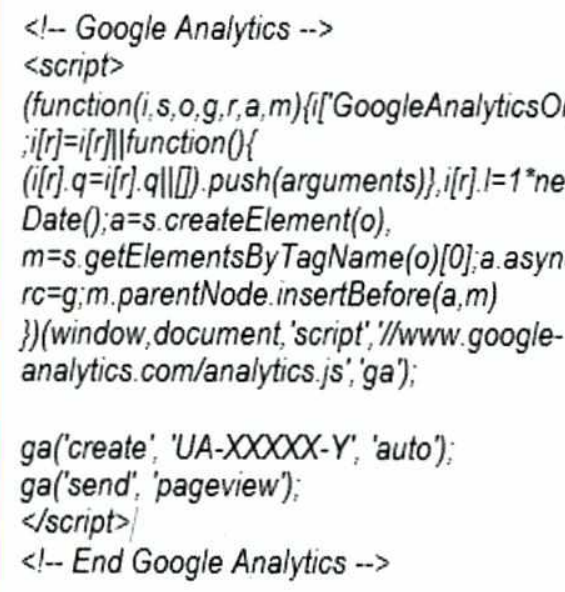

Fuente: Los autores

Es necesario recalcar que en la cadena 'UA-XXXXX-Y' se reemplazó el código de seguimiento brindado por la herramienta Google Analytics. El código está estructurado por cuatro eventos específicos.

El primer evento corresponde a crear un <script> lo que lleva a descarga de la librería Analytics.js de JavaScript. El segundo, corresponde a la inicialización de la función ga global, esta función brinda la posibilidad de programar la ejecución de comandos. El tercer evento, agrega un comando a la cola de los comandos de la función ga, para permitir la creación de un objeto de seguimiento por medio del código de seguimiento generado. Finalmente, el cuarto evento, corresponde a la agregación de otro comando a la cola de comandos de la función llamada ga, con el propósito de 
enviar una página que fue vista en Google Analytics.

\section{¿Cómo funciona analytics.js?}

Primeramente, el código de seguimiento define una función llamada ga, esta es una función global que se denomina cola de comandos, porque agrega los comandos entrantes a la cola en lugar de ejecutarlos inmediatamente, hasta que la librería sea cargada completamente. Posteriormente, previo a que se cargue la librería analytics.js, se realiza una llamada a la función ga, para posteriormente en el final de la matriz q, adjuntar la lista de argumentos enviados a la función. Una vez que la biblioteca Analytics.js se ha cargado se inspecciona el contenido de la matriz ga.q y ejecuta cada comando por orden.

En el caso de agregar comandos a la cola, el primer comando o parámetro identifica un método de analytics.js mediante una cadena. Un método global como create hace referencia mediante un determinado comando. Por otro lado, el método send puede ser un método de instancia de seguimiento.

A continuación se pueden observar dos comandos del fragmento de seguimiento: ga('create', 'UA-XXXXX-Y', 'auto'); ga ('send','pageview'). En el comando create, se reciben los campos trackingId, cookie Domain y name que corresponden a los parámetros segundo, tercero y cuarto, respectivamente. Por otro lado, el comando send acepta un segundo parámetro hit Type opcional.

Todos los comandos aceptan un parámetro fieldsObject final que también pueden ser especificados para utilizar otros campos.

Marek (2011) menciona que Google
Analytics requiere la utilización de servidores de terceros para su funcionamiento, en este caso, los servidores de Google. Su conexión requiere una cuenta de Gmail para acceder al sitio oficial de Google Analytics en la siguiente URL http://www.google.com.ec/intl/es/ analytics/.

Para este análisis, se ingresó con los datos de la cuenta de Gmail y se procedió a la conexión del sitio web. Sin embargo, para la conexión del sitio se requirió información como: el nombre de una cuenta, el nombre del sitio web, el URL del sitio web, la categoría o sector de trabajo y por último, la zona horaria.

Finalmente, Google Analytics generó un código de seguimiento para empezar el análisis del tráfico web, este código fue insertado en las páginas web del análisis dentro de las etiquetas $<$ header $></$ header $>$ $0<$ footer $><$ /footer $>$

\section{b. Reporte.}

Las herramientas Piwik y Google Analytics generaron gráficos de visitantes en la página web, haciendo visibles resultados sobre aspectos como: número de visitantes únicos, tiempo de duración de visitas, porcentaje de tasas de rebote, secciones de las páginas más visitadas, procedencia de las visitas, tipos de sistemas operativos usados, o dispositivos mediante los que accedieron a la página web (teléfonos móviles, tabletas, portátiles o computadoras de escritorio).

En la Figura 6 se puede observar una captura de la pantalla del dashboard (gráfico de las métricas que intervienen en el logro de los objetivos de la estrategia) utilizado por Piwik y en la Figura 7, se muestra parte del dashboard de Google Analytics. 


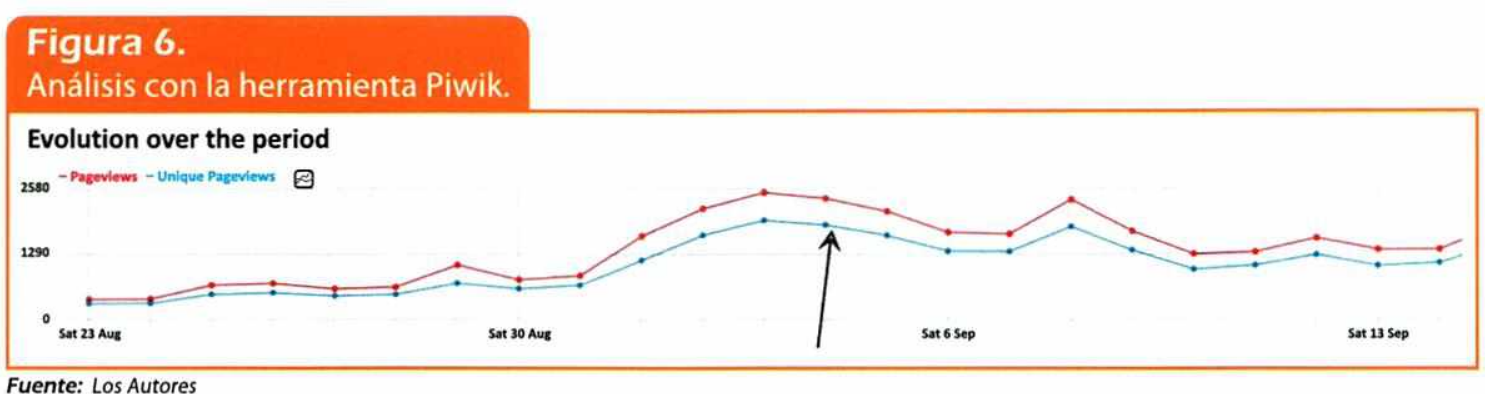

\section{Figura 7.}

Análisis con la herramienta Google Analytics.

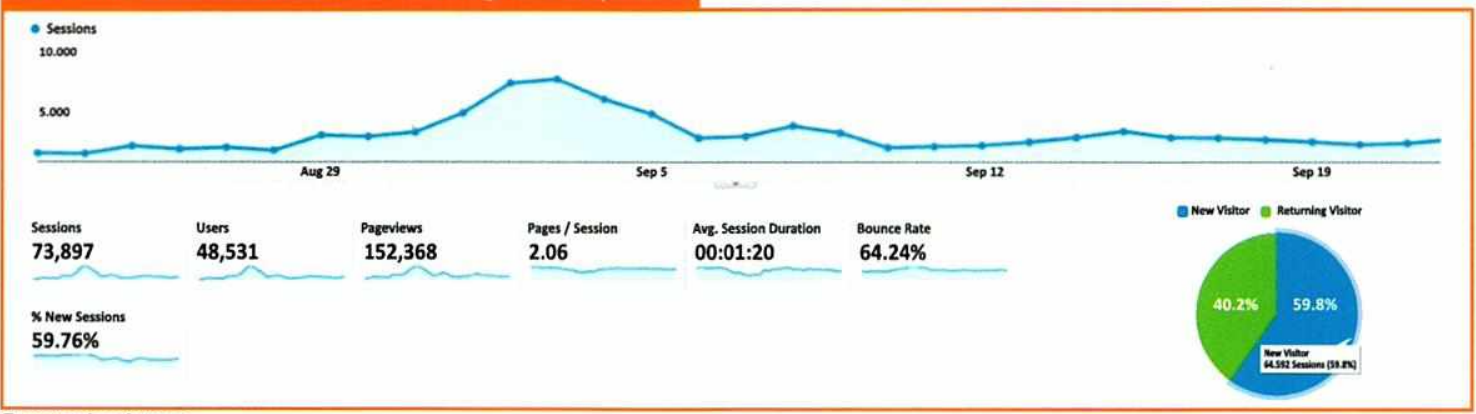

Fuente: Los Autores

\section{d. Análisis.}

En la Tabla 2 se puede observar los resultados obtenidos con la implementación de las métricas propuestas en el caso de las redes sociales. Para esto, se contrastaron los "Datos Base" con los que se inició el análisis, contra los "Datos Actuales" que fueron los obtenidos al finalizar este estudio. Los porcentajes devueltos ayudaron a entender el comportamiento de los usuarios y la interacción de las redes con el negocio de la escuela de postgrado del Ecuador.

Con los datos obtenidos, luego de la implementación de la estrategia de marketing digital, se evidenció que las interacciones de los usuarios se incrementaron en Facebook en un $75.21 \%$ y en Twitter un $90.68 \%$ sobre comentarios y menciones mostrando una mayor interacción de los usuarios. Es necesario indicar que Twitter es la red social que obtuvo más acogida.

\begin{tabular}{|c|c|c|c|c|c|}
\hline $\begin{array}{l}\text { Tabla } \\
\text { Result }\end{array}$ & do del anális & en las & es socia & & \\
\hline An & isis redes sociales & & Base & & tuales \\
\hline & & $f$ & 5 & $f$ & $y$ \\
\hline Int & acciones & 29 & 15 & 117 & 161 \\
\hline & & 45 & 61 & 97 & 113 \\
\hline & & 71 & 53 & 107 & 117 \\
\hline $\mathbf{A u}$ & iencia & 2962 & 3120 & 3500 & 4359 \\
\hline & & 149 & 160 & 331 & 397 \\
\hline & Conversación & $0.98 \%$ & $0.48 \%$ & $3.34 \%$ & $3.69 \%$ \\
\hline.$\stackrel{n}{E}$ & Amplificación & $1.52 \%$ & $1.96 \%$ & $2.77 \%$ & $2.6 \%$ \\
\hline 市 & Acción & & $1.7 \%$ & & $2.7 \%$ \\
\hline हे & Aceptación & $2.4 \%$ & & $3.1 \%$ & \\
\hline ن & Interacciones & $4.9 \%$ & $2.43 \%$ & $9.2 \%$ & $6.3 \%$ \\
\hline & Conversación & $19.46 \%$ & $9.38 \%$ & $35.35 \%$ & $40.56 \%$ \\
\hline & Amplificación & $1.52 \%$ & $1.96 \%$ & $2.77 \%$ & $2.59 \%$ \\
\hline 苞 & Acción & & $38.1 \%$ & & $28.5 \%$ \\
\hline$\Xi$ & Aceptación & $47.7 \%$ & & $32.3 \%$ & \\
\hline & Interacciones & $97.3 \%$ & $47.5 \%$ & $97 \%$ & $69.0 \%$ \\
\hline
\end{tabular}

Fuente: Los autores 
$\mathrm{Al}$ analizar por medio del indicador las posibles interacciones de publicaciones de la escuela de postgrado del Ecuador con personas por medio de las redes sociales, se obtuvo en Facebook el $53.61 \%$ y en Twitter el $46.02 \%$. En las métricas referentes a la audiencia se destaca un incremento de Fans en Facebook en un $15.37 \%$ de usuarios, en el caso de Twitter en un $28.42 \%$, su incremento, lo que ayuda a aumentar el número de nuevos posibles clientes.

En el análisis de -Compromiso- los resultados del número de comentarios sobre fans se incrementaron en un $2.36 \%$; el número de publicaciones sobre el número de fans en un $1.25 \%$; el número de "Me gusta" sobre fans, en un $0.70 \%$ y finalmente, el número de comentarios sumando las publicaciones compartidas y las opciones "Me gusta", sobre el número de fans, se incrementó en un $4.30 \%$. Estos datos en la red social Facebook.

Los resultados en el análisis del compromiso de los usuarios hacia el negocio en la red social Twitter, indicaron que el número de menciones sobre seguidores, tuvo un incremento del 3.2\%; el número de ReTweet sobre el número de seguidores, un incremento del $0.6 \%$; el número de Clics en enlaces sobre seguidores, un incremento del $1.0 \%$ y finalmente, el número de Menciones sumado los ReTweet sobre el número de seguidores, en un $3.9 \%$.

El análisis de -Interés- indica que el número de comentarios sobre publicaciones se incrementó el $15.89 \%$; el número de publicaciones compartidas sobre el número de publicaciones, en el 1.25\%; el número de “"Me gusta"” sobre publicaciones, un decremento del $15.40 \%$ y finalmente, el número de comentarios sumado las publicaciones compartidas y la opción "Me gusta" sobre el número de publicaciones, disminuyó en un $0.30 \%$ : Estos datos en la red social Facebook.

En el caso de la red social Twitter, indica que el número de menciones sobre Tweet se incrementó en el 31.2\%; el número de ReTweet sobre el número de Tweet, en un $0.6 \%$; el número de Clics en enlaces sobre Tweet; disminuyó en un $9.6 \%$; finalmente, el número de Menciones sumado los ReTweet sobre el número de Tweets se incrementó en un $21.5 \%$.

Una vez analizado el comportamiento en las redes sociales, se procedió al análisis de cuatro objetivos que se esperaban obtener con la implementación de una estrategia digital, para la escuela de postgrado del Ecuador.

En el primer objetivo Dar respuesta a clientes ofreciendo los servicios, presentado en la Tabla 3, la métrica referente a la tasa de rebote indica un porcentaje del $23.1 \%$, en el periodo de estudio se logró un decremento de la tasa de rebote del $53.82 \%$, lo que refleja que los usuarios están interesados en conocer las maestrías ofertadas por la escuela de postgrado y acceden al sitio web.

\begin{tabular}{|c|c|c|c|c|}
\hline \multicolumn{5}{|c|}{ Resultados del objetivo "Dar respuesta a clientes ofreciendo los servicios". } \\
\hline $\begin{array}{l}\text { Análisis del } \\
\text { objetivo }\end{array}$ & Métricas & Base & Google Analytics & Piwik \\
\hline \multirow{4}{*}{ 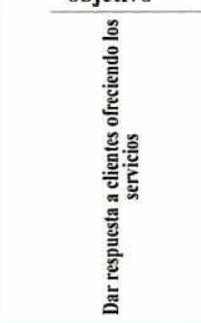 } & $\begin{array}{l}\text { Tasa de rebote } \\
\text { (Visitas < a } 15 \mathrm{~s})\end{array}$ & $76.92 \%$ & $23.1 \%$ & $23.1 \%$ \\
\hline & $\begin{array}{l}\text { Tiempo de conversión } \\
\frac{\text { Conversiones }}{\text { visitas }}\end{array}$ & $2.67 \%$ & $5.96 \%$ & $5.95 \%$ \\
\hline & $\begin{array}{l}\text { Tiempo de visita } \\
\text { Páginas vistas } \\
\text { visitas }\end{array}$ & $17.4 \%$ & $44.28 \%$ & $44.28 \%$ \\
\hline & $\begin{array}{c}\text { visitas } \\
\text { Buscador interno } \\
\text { (Análisis de palabras clave) }\end{array}$ & $22.7 \%$ & $33.72 \%$ & $33.72 \%$ \\
\hline
\end{tabular}


El tiempo de conversión corresponde al número de usuarios que hicieron contacto sobre el número total de visitas obteniendo un incremento del $3.29 \%$, lo que significa que los usuarios llenaron los formularios de contacto para obtener más información sobre las maestrías. Otra métrica de análisis es el tiempo de visita, conseguido del número de páginas vistas sobre las visitas, se obtuvo un incremento del $26.88 \%$, que significa que la información presentada es interesante para los usuarios. Este incremento fue producido en las páginas de cada una de las maestrías ofertadas.

La Tabla 4 que corresponde al objetivo Vender el producto presenta que la métrica de permanencia en el sitio se incrementó en un $30.6 \%$, lo que indica que los usuarios visitaron cada una de las páginas ofertadas en la estrategia digital, estas contienen información sobre las maestrías y formularios de contactos.

Finalmente, el análisis del tráfico de visitas se lo hizo en base a los motores de búsqueda, como son: Google Chrome, Firefox, Safari, entre otros. El porcentaje de esta métrica se redujo en un $18.43 \%$, es un indicador de mucha prioridad, debido a que la estrategia se centraba en las redes sociales, poniendo así en evidencia la eficacia de una estrategia de marketing digital.

\begin{tabular}{|c|c|c|c|c|}
\hline \multicolumn{5}{|c|}{ Resultados del objetivo "Vender el producto". } \\
\hline $\begin{array}{c}\text { Análisis } \\
\text { del } \\
\text { objetivo }\end{array}$ & Métricas & Base & $\begin{array}{l}\text { Google } \\
\text { Analytics }\end{array}$ & Piwik \\
\hline \multirow{3}{*}{ 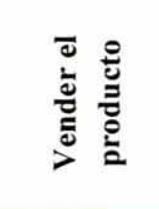 } & Permanencia en el sitio (Número de páginas vistas) & $40.7 \%$ & $71.3 \%$ & $71.3 \%$ \\
\hline & $\begin{array}{c}\text { Tráfico } \\
\text { Visitantes desde motores de búsqueda }\end{array}$ & $21.4 \%$ & $2.97 \%$ & $2.97 \%$ \\
\hline & $\begin{array}{c}\text { Visitantes } \\
\end{array}$ & & & \\
\hline
\end{tabular}

Fuente: Los autores

La Tabla 5 que muestra el tercer objetivo Incrementar y mantener la fidelidad del lector, está compuesta por las métricas de análisis de contenido que se obtiene de páginas vistas sobre visitas a contenidos, esta métrica se incrementó en un $53.29 \%$ mostrando que la información publicada es interesante para los usuarios. La métrica para calcular el porcentaje de usuarios fieles se la realizó dividendo el número de vistas nuevas, sobre el número de visitas recurrentes, obteniendo un incremento del $8.38 \%$ de usuarios que volvieron a visitar el sitio web.

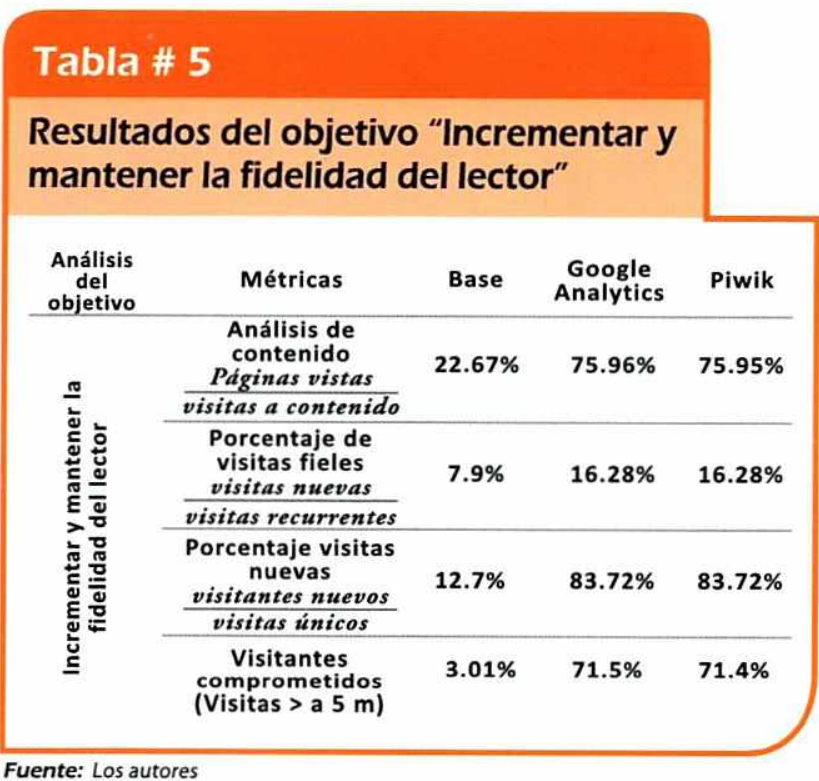


Como se evidencia en la Tabla 5, una de las métricas más importantes fue el porcentaje de visitas nuevas, o usuarios nuevos del sitio obtenido, mediante el número de visitantes nuevos, sobre el número de visitantes únicos, dando como resultado un incremento del $71.02 \%$ de nuevos usuarios en el sitio, demostrando la efectividad de una campaña en las redes sociales.

La Tabla 6 del cuarto objetivo Conseguir posicionamiento de la marca en la web y el interés por usuario, se analiza la tasa de conversión obtenida por el número de visitas al formulario de contacto, sobre las visitas, obteniendo un incremento del $16 \%$ en las solicitudes de más información.

\section{Tabla \# 6 \\ Resultados del objetivo "Conseguir posicionamiento de la marca en la web y el interés por usuario}

Análisis

del

Métricas

objetivo

- Tasa de conversión

visitas al formulario de

Tiempo de permanecía en

(Secciones más visitadas)

Adquisición de tráfico visitas tráfico directo + visitas redes sociales contacto

$1.01 \%$

$17 \%$

$17.01 \%$

visitas

el sitio

$15.91 \%$

$91.3 \%$

$91.3 \%$

$9.4 \%$

$97.03 \%$

$97.07 \%$

\section{Google
Analytics}

Piwik

visitas

Fuente: Los autores

El tiempo de permanencia en el sitio, compuesta por el parámetro de secciones más visitadas, se incrementó en un $75.39 \%$, estas correspondieron a las páginas específicas de las maestrías. Otra de las métricas más importantes en el estudio fue la adquisición de tráfico, debido a que el estudio y la implementación de una estrategia de marketing digital, se centraba en las redes sociales, esta métrica se la obtiene de las visitas de trafico directo, sumado a las visitas de redes sociales, sobre el número total de visitas, obteniendo un $97.03 \%$ de visitas desde las redes sociales.

\section{e. Optimización.}

En esta etapa de la Analítica Web se establecieron parámetros para mejorar la interacción y captura de nuevos usuarios, actualizando el sitio con una plataforma de manejo de contenido web, como Word Press que está optimizado para que la página web aparezca como primera opcion en los motores de busqueda Google, Bing, Yahoo, entre otros.

\section{Conclusiones}

A través de esta investigación, se ha determinado que la herramienta de 
analítica web permite conocer las preferencias de los usuarios, tener los objetivos claros, monitorear indicadores claves y establecer una metodología de mejora continua. Todo esto se constituye en ingredientes perfectos para optimizar y rentabilizar cualquier negocio. El establecer las métricas apropiadas en el objeto de estudio, ayuda a plantear objetivos reales y medibles.

El planteamiento y estudio de una estrategia de marketing digital, ayuda a conocer el comportamiento y necesidades de los usuarios; de esta manera, las redes sociales, contribuyen en la visibilidad de la empresa, mejorando su presencia y reputación en internet, incluyendo las instituciones de educación superior.

Con Piwik la información se encuentra en un servidor local; es decir, que los datos son propios, a diferencia de Google Analytics que utilizan los servidores de Google. Si la información es de suma importancia, sensible y la propiedad y privacidad de los datos, son prioridad en la empresa, Piwik es la mejor opción a ser implementada.

La utilización de las herramientas Piwik y Google Analytics se pudieron obtener gráficos estadísticos que facilitan la lectura y análisis. Cabe destacar que en Piwik se dificultó el manejo de grandes volúmenes de datos, especialmente en la segmentación; a diferencia de Google Analytics, que facilitó el proceso de filtro de los datos recabados. A través de este estudio quedó evidenciado que se incrementaron las interacciones de los usuarios tanto en Facebook (75.21\%), como en Twitter $(90.68 \%)$ sobre menciones y comentarios, lo que se traduce como una mayor interacción de los usuarios. La tasa de rebote; es decir el porcentaje de visitas considerando el tiempo de permanecía en el objetivo: Dar respuesta a clientes ofreciendo los servicios, tuvo un decrecimiento del $53.82 \%$, lo que indica que los usuarios que accedieron a través de las redes sociales a la página web de la escuela de postgrado del Ecuador, demostraron interés en los programas académicos que oferta.

Mediante el objetivo: Vender el producto, se pudo establecer que hubo un decremento del $18.43 \%$, en el uso de los diferentes motores de búsqueda para acceder al sitio web de la escuela de postgrado del Ecuador. Esto ratifica la eficacia de la aplicación de una estrategia de marketing digital, apoyada en redes sociales como Facebook y Twitter.

\section{Al analizar el objetivo: Incrementar $y$} mantener la fidelidad del lector, fue posible observar que el uso apropiado de campañas en redes sociales soportadas en una estrategia de marketing digital debidamente estructurada, da resultados positivos en indicadores como el incremento de nuevos usuarios, que serían potenciales clientes. Así quedó demostrado en el presente estudio, donde se incrementó este indicador en un $71.02 \%$.

\section{El objetivo: Conseguir posicionamiento de} la marca en la web y el interés por usuario, permitió demostrar que la adquisición de tráfico, utilizando anuncios a través de las redes sociales, aumentó considerablemente el tráfico al sitio web de la escuela de postgrado. En este trabajo se evidencia, con el incremento de un $97.03 \%$ de visitas desde las redes sociales.

Así mismo, se evidencia al obtener mediciones fiables, que las herramientas de analítica web: Google Analytics y Piwik permitieron realizar un seguimiento de clientes efectivo de las estrategias de marketing digital. En sintesis, es importante que una vez que se haya conseguido incrementar el tráfico de usuarios en la página web, a través de las redes sociales, se integre una plataforma para manejo de clientes, para hacer un seguimiento apropiado y convertirlos en clientes reales. 


\section{Referencias bibliográficas}

Agüera, M. (2014). Analítica web y redes sociales. Herramientas de análisis. Recuperado a partir de http://repositorio. bib. upct.es:8080/dspace/handle/10317/4036.

Brunetta, H. (2013). Marketing digital ebook: Claves para implementar estrategias efectivas en redes sociales. RedUsers.

Caldevilla. (2010). Las Redes Sociales. Tipología, uso y consumo de las redes 2.0 en la sociedad digital actual. Universidad Complutense de Madrid.

Campos. (2008). Las redes sociales trastocan los modelos de los medios de comunicación tradicionales. Revista Latina de Comunicación Social.

Degiorgi, H., \& Méndez, A. (2013). Aplicación de indicadores para la evaluación de consulta de un repositorio Institucional. Presentado en II Taller de Indicadores de Evaluación de Bibliotecas (27 y 28 de junio del 2011, La Plata, Argentina). Recuperado a partir de http:/hdl.handle.net/10915/26823.

Digital Analytics Association. (2015). Recuperado el 28 de octubre del 2015, a partir de http://www.digitalanalyticsasso ciation.org/.

Fernández-Villavicencio, N., Menéndez, J., Catuxa Seoane García, C. \& San Millán, M. Revisión y propuesta de indicadores (KPI) de la Biblioteca en los medios sociales. Recuperado a partir de http://search. proquest.com/docview/1348600376? acc ountid $=35177$

Gálvez, I. G. (2010). Introducción al Marketing en Internet: Marketing 2.0. IC Editorial..

García, \& Aguado. (2009). Del Worl of mouth al marketing viral: aspectos claves de la comunicación a través de redes sociales. Comunicación y Hombre.
Hasan, L., Morris, A., \& Probets, S. (2009). Using Google Analytics to Evaluate the Usability of E-Commerce Sites. En M. Kurosu (Ed.), Human Centered Design (pp. 697-706). Springer Berlin Heidelberg. Recuperado a partir de http://link. springer.com/chapter/10.1007/978-3-642 -02806-9_81.

Hess, K. M. (2012). Discovering Digital Library User Behavior with Google Analytics. Recuperado a partir de https:// www.ideals.illinois.edu/handle/2142/ 35261.

Kotler, P., \& Armstrong, G. (2003). Fundamentos de marketing. Pearson Educación.

Lamas, C. (2015). TELOS - Fundación Teléfonica - Detalle Anteriores - Los medios interactivos y su publicidad. La medición de audiencias. Recuperado el 2 de diciembre de 2015, a partir de https://telos.fundaciontelefonica.com/se ccion $=1268 \&$ idioma $=$ es_ES $\&$ id $=201002$ 0409130001 \&activo $=6$.do.

Lebrón, C. M., \& M, C. (2011). Analítica web con Google Analytics. Bubok.

Maldonado, S. (2010). Analítica Web: medir para triunfar. ESIC Editorial.

Marek, K. (2011). Chapter 2: Getting to Know Web Analytics. Library Technology Reports, 47(5), 11-16.

Martínez, M. M. (2013). Analítica web para empresas: Arte, ingenio y anticipación. Editorial UOC.

Miller, S. A. (2012). Piwik Web Analytics Essentials. Packt Publishing Ltd.

Morales, M. M. (2010). Analítica Web para empresas. Editorial UOC.

Morley, S. (2015). Understanding the Google Analytics tracking code. Recuperado el 2 de diciembre del 2015, a partir de 
http://code.stephenmorley.org/java script/understanding-the-google-analyti cs-tracking-code/.

Olguín, L. A., \& Klenzi, R. O. (2012). Analítica web en centros de información. Presentado en XIV Workshop de Investigadores en Ciencias de la Computación. Recuperado a partir de http:/hdl.handle.net/10915/ 18684.

Olmo, J. L. del, \& Gascón, J. F. F. (2014). Marketing digital en la Moda. Ediciones Universidad de Navarra.

Pérez, F. (2013). La Comunicación, nuevas tendencias. Recuperado a partir de http://search.proquest.com/docview/150 8553596 ? accountid $=35177$

Piwik. (2015). Free Web Analytics Software. Recuperado el 28 de octubre del 2015, a partir de http://Piwik.org/.

Plaza. (2009). Monitoring web traffic source effectiveness with Google Analytics: An experiment with time series. Aslib Proceedings, 61(5), 474-482. http://doi. org/10.1108/00012530910989625.

Philip Kotler y Gary Armstrong, en "Fundamentos de Marketing", $8^{\mathrm{a}}$ edición, 2008.

Sanjaime. (2012). Redes Sociales y Marketing. Universidad Politécnica de Valencia.

Sheehan, B. (2010). Basics Marketing 02: Online Marketing. AVA Publishing.

Uribe. (2010). Uso de las redes sociales como herramienta de marketing. Estudio de casos.

Villaplana, R. (2013a, agosto 9). Analítica Web: Métricas imprescindibles para optimizar tu estrategia SEO. Recuperado el 2 de diciembre del 2015, a partir de http://www.cloud-tic.com/ analiticaweb-metricas-imprescindibles-para-opti mizar-tu-estrategia-seo/.

Villaplana, R. (2013b, diciembre 16). Redes Sociales: Cómo medir la popularidad de una campaña en Social Media. Recuperado el 2 de diciembre del 2015, a partir de http://www.cloud-tic.com/ redes-sociales-como-medir-la-populari dad-de-una-campana-en-social-media/.

Villaplana, R. (2014, mayo 8). ¿Qué metodología seguir para una Analítica Web eficaz? Recuperado el 2 de diciembre del 2015, a partir de http://www.cloudtic.com/metodologia-seguir-para-una-a nalitica-web-eficaz/.

\section{Vilma Álvarez Intriago}

Ingeniera en Computación.

Magister en Administración de Empresas.

Docente de la Facultad de Sistemas, Telecomu-

nicaciones y Electrónica de la Universidad Espíritu Santo - Ecuador.

E-mail: vkalvarez@uees.edu.ec

\section{Luis Agreda Fernández}

Estudiante de la Carrera de Sistemas.

Facultad de Sistemas, Telecomunicaciones y Electrónica. Universidad Espíritu Santo - Ecuador.

E-mail: leagreda@uees.edu.ec

\section{Antonio Cevallos Gamboa}

Ingeniero en Sistemas.

Magister en Administración de Empresas.

Magister en Sistemas de Información Gerencial

$\mathrm{PhD}$ (c) en Ciencias de la Dirección

Decano Facultad de Sistemas, Telecomuni-

caciones y Electrónica. Universidad Espíritu Santo - Ecuador

E-mail: acevallos@uees.edu.ec 
